\title{
Iatrogenic heart block during treatment of a patient with Cushing's syndrome: report of a case
}

\author{
J. P. Brito $\cdot$ E. Singh $\cdot$ A. Basu
}

Published online: 29 November 2011

(C) Springer Science+Business Media, LLC 2011

An 83-year-old woman with history of hypertension treated chronically with verapamil $360 \mathrm{mg}$ daily, presented with refractory hypokalemia and hypochloremic alkalosis. Initial physical examination demonstrated mild hypertension with proximal muscle weakness and normal cardiovascular exam.

Initial workup revealed an a.m. cortisol level of $70 \mathrm{mcg} /$ dl (6-23), a 24-h urine-free cortisol of 5,719 $\mu \mathrm{g}(10-100)$, $1 \mathrm{mg}$ overnight dexamethasone suppression test that showed a morning cortisol level of $54.6 \mu \mathrm{g} / \mathrm{dl}$, and ACTH level of $123 \mathrm{pg} / \mathrm{ml}(6-58)$.

A diagnosis of ACTH-dependent Cushing's syndrome, probably of ectopic origin, was made. While undergoing further evaluation, she was administered $300 \mathrm{mg}$ of ketoconazole thrice a day orally, and 2 days later, the dosage was increased to $300 \mathrm{mg}$ every $6 \mathrm{~h}$. Hypokalemia was corrected during the first $24 \mathrm{~h}$ after admission with oral potassium. On the third day, the patient developed severe hypotension $(79 / 38 \mathrm{mmHg}$ ), bradycardia (38 beats per minute), and altered mental status. Electrocardiogram showed complete heart block with a slow junctional escape rhythm, and electrolyte panel did not show any abnormality. She was not responsive to intravenous atropine, and thus, she was transferred to coronary care unit where a transvenous temporary pacemaker was placed. Verapamil and ketoconazole were discontinued immediately. Of note, medication reconciliation and past medical history were reviewed and no other drug-drug interaction or prior history or any arrhythmia was found.

J. P. Brito $(\bowtie) \cdot$ E. Singh · A. Basu

Division of Endocrinology, Diabetes, Metabolism and Nutrition, Mayo Clinic, 200 First Street SW, Rochester, MN 55905, USA e-mail: Juan.brito@mayo.edu
The next day, the patient recovered completely, the ECG normalized, and the pacemaker was removed.

Comment: Ketoconazole is an adrenal enzyme inhibitor that blocks the first step of cortisol biosynthesis [1]. It is also a CYP3A4 inhibitor [2]. Hypertension is a presenting feature of Cushing's syndrome and requires, not infrequently, high doses of antihypertensives to achieve blood pressure control. Verapamil is also a CYP3A4-based inhibitor and is classified as a moderate CYP3A4 inhibitor in the FDA guidance [3]. In our patient, ketoconazole might have increased the levels of verapamil in the blood to toxic levels and caused the heart block. To our knowledge, verapamil-ketoconazole interaction has not been reported previously, probably because common uses of ketoconazole, such as to treat fungal infections, require lower doses than those necessary for Cushing's syndrome. The case strongly suggests a possible interaction between the two drugs.

\section{References}

1. G.K. Stalla, J. Stalla, M. Huber et al., Ketoconazole inhibits corticotropic cell function in vitro. Endocrinology 122(2), 618 (1988)

2. T.D. Bjornsson, J.T. Callaghan, H.F. Einolf et al., The conduct of vitro and in vivo drug-drug interaction studies: a Pharmaceutical Research and Manufactures of America (PhRMA) perspective. Drug Metab. Dispos. 31(7), 815-832 (2003)

3. Y.Y. Wang, R. Jones, S. Hall, Differential mechanism-based inhibition of CYP3A4 and CYP3A5 by verapamil. Drug Metab. Dispos. 33(5), 664-671 (2005) 